\title{
Estudios sobre la Mujer en Tres Revistas de Salud Pública
}

\section{Studies on Women in Three Public Health Journals: A Bibliographical Review}

\section{Cristina A. Montero'}

MONTERO, C. A. Studies on Women in Three Public Health Journals: A Bibliographical

Review. Cad Saúde Públ, Rio de Janeiro, 11 (2): 305-313, Apr/Jun, 1995.

The purpose of this study is determine how the First National Conference on Women's Health Rights, held in Brasilia in 1986, was reflected in three main Brazilian public health journals. The articles were published in Revista de Saúde Pública (Faculdade de Saúde Pública, Universidade de São Paulo), Saúde em Debate (Centro Brasileiro de Estudos de Saúde) and Cadernos de Saúde Pública (Escola Nacional de Saúde Pública, Fundação Oswaldo Cruz), from 1986 to 1993.

Key words: Women Health; Public Health; Scientific Publications

\section{INTRODUCCION}

Para que nuestras voces sean escuchadas, debemos unir nuestras reflexiones $y$ descubrimientos, mostrando nuestra parte de la historia. La humanidad comenzó a percibir el error histórico cometido al ignorar, por tanto tiempo, la «visión de mundo» de las mujeres. Esto nos hizo pensar en investigar las publicaciones sobre la mujer presentes en la literatura de salud pública en el contexto brasileño, observar cómo son abordados los diferentes temas que se relacionan con su salud, y otros aspectos que cruzan y definen su existencia y cotidianidad.

Los últimos años han sido ricos en esta discusión, en el seno de las organizaciones feministas, gremiales, políticas y académicas; la sociedad comenzó a tener interés por conocer más sobre la mujer.

En el año 1983 se formula en Brasil el "Programa de Assistencia Integral á Saúde da Mulher" (PAISM), con el objetivo de dar una atención diferenciada a la población femenina a partir de una acción educativa y de

\footnotetext{
${ }^{\prime}$ Centro de Estudos de Saúde do Trabalhador e Ecologia Humana, Escola Nacional de Saúde Pública, Fundação Oswaldo Cruz, Rua Leopoldo Bulhões, 1480, Rio de Janeiro, RJ, 21041-210, Brasil.
}

intervención para promover la recuperación de la salud con la participación de las usuarias y profesionales del área (MS/INAN, 1983). En esta propuesta, la idea de tratar la mujer como ser integral, con ciclos de vida que tienen fases en las cuales profundas modificaciones existen y que deben tener asistencia especial, así como el reconocimiento de que estas fases son pasos naturales en la vida de las mujeres y que orientación y apoyo de los servicios de salud no significan su medicalización o el tratamiento como patologías (CNDM, 1986).

En medio de esta nueva experiencia y reflexiones, sumadas a las prácticas de grupos no gubernamentales, se realiza en Brasília la Conferencia Nacional de Salud y Derechos de la Mujer, del 10 al 13 de octubre de 1986, creando la oportunidad para debatir diferentes aspectos en relación a la mujer, a partir de ponencias que discutían sobre su identidad de acuerdo a raza, edad, ocupación, sexualidad, derechos reproductivos y anticoncepción, aspectos relacionados con los servicios de salud, relación entre la salud y el trabajo, salud mental, relación médico/paciente, salud y ciudadanía, así como la discusión sobre los derechos de la mujer. Es importante ubicar este marco para entender lo que se pretende con esta revisión. Se trata de ver como todas estas experiencias, análisis y debates que se han 
dado sobre la mujer, pernearon y se hicieron presentes en la producción académica de salud pública a partir de los artículos que incluyeron la problemática de la mujer y fueron publicados durante el tiempo transcurrido desde el año en que se realizó la Conferencia hasta el primer semestre del año 1993. La introducción sobre esta discusión en el seno de las ciencias sociales y en especial de la salud pública no ha sido fácil, al menos desde una perspectiva que trate de los aspectos históricos, ideológicos, políticos e institucionales, y con una metodología acorde con estos objetivos. Labra (1989: 17-18) señaló esta preocupación diciendo " $O$ aspecto sui generis da discussão do tema saúde da mulher é que ele surge, se enriquece e se alarga fora do setor saúde, ou seja, no próprio seio da sociedade civil. Quer dizer, a vanguarda do movimento de mudança das políticas de saúde dirigidas á mulher não foi constituída por segmentos progressistas da burocracia estatal, e sim por lideranças de organizações de mulheres empenhadas em levar para o interior daquelas suas questões específicas".

\section{OBJETIVOS}

- Observar la influencia que tuvo la Conferencia Nacional de Salud y Derechos de la Mujer, realizada en Brasília en el año 1986, sobre las publicaciones del área de la Salud Pública.

- Específicamente mostrar los artículos publicados sobre el tema de la mujer en las revistas Cadernos de Saúde Pública, Revista de Saúde Pública, Saúde em Debate, en el periodo de 1986 a 1993. Organizando los mismos por áreas temáticas.

- Dar una visión amplia sobre las líneas editoriales en esta área, sin pretender realizar un análisis profundo sobre el contenido y metodología presentes en estas publicaciones, y sí una idea global sobre los principales aspectos tratados en estos trabajos.

\section{MATERIALESY METODOS}

Se escogieron tres de las principales revistas brasileñas en el área de la salud pública, que representan centros de estudios e investigación en el área, grupos de opinión y profesionales de la salud pública a nivel nacional. Fueron seleccionados todos los artículos relacionados con la mujer, publicados durante el periodo comprendido entre 1986 y 1993 (primer trimestre).

Se tomaron las ponencias presentadas en la Conferencia Nacional de Salud y Derechos de la Mujer (Brasilia, 1986), y se agruparon por temas de discusión. Empleamos esta misma agrupación para los artículos publicados por las revistas como referencia en el análisis. Esta agrupación será presentada más adelante. Se incluyeron las revistas:

- Cadernos de Saúde Pública. Escuela Nacional de Salud Pública. Fundación Oswaldo Cruz (ENSP/FIOCRUZ).

- Revista de Saúde Pública. Facultad de Salud Pública. Universidad de SãoPaulo (FSP/USP).

- Saúde em Debate. Londrina. Centro Brasileño de Estudios de Salud (CEBES).

Fueron revisados todos los números publicados a partir del año 1986, que se encontraban disponibles en la biblioteca de la ENSP/ROCRUZ.

De los Cadernos de Saúde Pública revisamos 27 números, de un total de 29 que se editaron durante el período 1986-1993; en éstos fueron publicados 16 artículos referidos a temas vinculados con la situación de la mujer. Es importante señalar que, del total de artículos, 10 aparecieron en el volumen 7 , número 2, número temático denominado "Mulher e Saúde", coordinado por Karen Mary Giffin. Es decir, en los restantes 26 números, sólo se publicaron seis artículos sobre el tema. La Revista de Saúde Pública publicó, en este tiempo, 44 números, de los cuales examinamos 40 . Se encontraron 18 artículos que se referían al tema investigado. De la revista Saúde em Debate faltaron tres revistas, que no se encontraban en la 
biblioteca de la ENSP, y, en todas las examinadas, sólo se encontró un artículo referente al tema de la mujer.

En total, entre las tres revistas, se reunieron 35 artículos, que se organizaron por temas según el material disponible. Los grupos formados fueron:

Mortalidad y morbilidad . 9 artículos,

Planificación familiar,

anticoncepción y

derechos reproductivos

7 artículos,

Embarazo, parto, puerperio,

aborto

11 artículos,

Servicios de salud,

relación médico/paciente . . . . 4 artículos,

Salud y trabajo . . . . . . 2 artículos,

Otros .

2 artículos.

La discusión respecto al contenido de estos trabajos se relacionó, cuando fue posible, con las ponencias presentadas en la Conferencia de Brasília, pretendiendo sólo mostrar el tipo de estudios que se publicaron y los temas abordados por éstos, sin llegar a un análisis profundo respecto a su contenido.

\section{ORGANIZACION POR AREA TEMATICA}

En esta parte presentaremos los artículos publicados, agrupando los textos por la afinidad de los temas tratados en ellos, formando lo que llamamos de áreas temáticas. La selección de los grupos dependió del material disponible, buscando correspondencia con los temas discutidos en la conferencia.

\section{Morbilidad y Mortalidad Femenina}

\section{"La morbilidad y mortalidad femenina tiene dos características: una específicamente femenina relacionada con los procesos reproductivos y otra que podría considerarse dentro de las enfermedades comunes y/o proceso de salud/enfermedad pero que en el caso de las mujeres adquiere una dimensión particular por las condiciones económicas, culturales y sociales en que nacen, crecen, se desarrollan y mueren”. (Ravelo, 1990: 34)}

En estos años se publicaron en las revistas estudiadas 9 artículos que trataron sobre la morbilidad y mortalidad femenina en Brasil. El primero, un estudio sobre mortalidad realizado en diez capitales brasileñas el año 1985, con tasas generales y específicas por cinco principales grupos de causa según sexo y estandarizados por edad, buscando comparar la mortalidad femenina y masculina por grupos de edad realizado por Aquino et al. (1991). Carvalheiro \& Mano (1992) estudiaron la mortalidad en la mujer de 15 a 49 años en el período 1985 a 1989 y la compararon con los datos de 1970 a 1974 en el municipio de Ribeirão Preto, São Paulo (Brasil), según procedencia, estado civil, siete grupos etarios y causas, según la Clasificación Internacional de Enfermedades.

Silva (1992) identificó el perfil epidemiológico de la mortalidad materna en el estado de Rio de Janeiro en el período 1977-88, la tasa de mortalidad y su tendencia temporal, su distribución por edades, grupos de causas y por local de ocurrencia, comparándolo con otros estados y países. Lolio et al. (1991) presentan las principales causas de muerte en mujeres de edad fértil en el municipio de SãoPaulo durante el año 1986 y las comparan con los resultados de la década de 1960, centrando su estudio en las diferentes causas cardiovasculares. Feitosa et al. (1991) realizaron un estudio retrospectivo de mortalidad materna por cardiopatía desde 1979-1989 hasta en la Escuela Paulista de Medicina.

Sobre morbilidad, fueron publicados cuatro artículos. Uno de ellos observa la morbimortalidad por cáncer durante el año 1986 realizado en seis estados brasileños por Gulnar \& Mendoça (1993). Otro trabajo compara morbilidad y uso de los Servicios de Salud por sexo y edad a partir de los datos obtenidos en la "Pesquisa Nacional por Amostra de Domicílios" (Aquino et al., 1992). En el municipio de Rio de Janeiro, Koifman et al. (1991) realizaron un estudio donde muestran las principales características epidemiológicas descriptivas de la distribución del SIDA en mujeres en la década de los 80. Hardy et al. (1990), a través de diagnósticos anatomopatológicos observaron el riesgo de 
enfermedad benigna de mama y las diferentes variables reproductivas en el Centro de Controle de las Enfermedades MaternoInfantiles de Campinas.

\section{Planificación Familiar, Anticoncepción y Derechos Reproductivos}

Es este, tal vez, uno de los aspectos más polémicos, cuando se discute sobre la salud de la mujer. Agrupamos aquí 7 de los artículos publicados. Sobre los diferentes aspectos relacionados con la planificación familiar, anticoncepción y derechos reproductivos, se pronunciaron los ponentes durante la Conferencia de Salud y Derechos de la Mujer, a través de los trabajos de Garcia (1986), Reis (1986) y otro trabajo con la posición del Ministerio de la Salud.

En estos años se publicaron siete artículos al respecto. Bronfman \& Castro (1989) ven la planificación familiar como política social, que se expresa como política poblacional o como política de salud donde existe un acuerdo entre los administradores y beneficiarios del programa que debería permitir su fácil implantación. Sin embargo, atribuyen el fracaso de este programa, en América Latina, al desfase entre las prácticas concretas de la planificación familiar, la implementación y el discurso que la legítima. Muestran casos de aplicación de los diferentes métodos anticonceptivos, en especial los de esterilización quirúrgica, donde predomina el autoritarismo y coerción para su implantación.

Costa et al. (1989) analizaron las prácticas contraceptivas utilizadas por mujeres de siete «favelas» de Rio de Janeiro, mostrando la prevalencia del uso de anticonceptivos, conocimiento, forma de obtención y experiencia en el uso de estos métodos. Schor \& Lopez (1990), a partir de historias médicas y entrevistas con adolescentes puérperas, en São Paulo, estudiaron el uso, conocimiento y fuentes de información, medios de adquisición, control y su relación con edad, escolaridad y estado marital. Koifman et al. (1987) evaluaron el resultado del método Norplant (método anticonceptivo baseado en la implantación hormonal intradérmico), en el Municipio de Rio de Janeiro, y analizaron los riesgos para la salud de las usuarias, así como los efectos encontrados después del abandono de éste.

La píldora anticonceptiva es el más frecuente método utilizado por las mujeres, ya sea en forma automedicada o con consulta médica. Hardy et al. (1991) entrevistaron mujeres de 15-49 años de edad que viven en un barrio pobre en el área metropolitana de São Paulo, estudiando la prevalencia, y asociación entre algunas características sociodemográficas de las usuarias, y la presencia, o no, de factores asociados a uso, diferencias entre las usuarias, y no usuarias, de los servicios de salud.

Boruchovitch (1992) realizó una revisión de la literatura científica, mostrando los diferentes factores que se han asociado al no uso de anticonceptivos en la adolescencia. Procuró integrar los resultados, preocupada tantó con el riesgo de embarazo no deseado como con el de SIDA, con miras a desarrollar una educación sexual más efectiva y realista en este grupo. En la conferencia Takiuti (1986) introdujo la discusión sobre la problemática de la mujer adolescente.

Huttly et al. (1990) entrevistaron mujeres en el período posterior al parto en el hospital. Cuatro años después, $80 \%$ de ellas fueron entrevistadas nuevamente en sus hogares. Observaron la presencia de embarazos posteriores y su relación con edad materna, escolaridad, renta familiar y el deseo o no, de nuevos embarazos.

\section{Embarazo, Parto, Puerperio y Aborto}

El embarazo, la asistencia prenatal, tipo de parto, el puerperio, así como sus consecuencias perinatales son aspectos tratados en los trabajos de Lopez et al. (1989), efectuado en un servicio de obstetricia de São Paulo con la población adolescente. Tanaka et al. (1989) establece relación entre estos parámetros con la mortalidad materna y perinatal, a partir de los datos oficiales para el Estado de São Paulo. Lippi et al. (1989) compara las condiciones del embarazo con el bajo peso al nacer. Siqueira et al. (1986) relacionaron el hábito de fumar con el peso del recién nacido. Bettiol et al. (1992) estudiaron 
estos aspectos en madres adolescentes. Guerra et al. (1990) estudiaron gestantes de primera consulta en el Municipio de SãoPaulo e investigaron la prevalencia de anemia de acuerdo al tiempo de gestación y número de partos anteriores.

Faundes \& Cecatti (1991) observaron los altos niveles de incidencia de cesáreas practicadas en Brasil y estudiaron su relación con los factores socio-culturales, de organización de la atención obstétrica, factores institucionales y legales, asociación con la esterilización quirúrgica y sus consecuencias sobre la mortalidad materna y perinatal.

Respecto al aborto se publicaron durante este periodo cuatro artículos que muestran la alta frecuencia de esta práctica, sus complicaciones, condiciones en las cuales se realizan, así como las diversas motivaciones, relación con estado civil, edad y uso de anticonceptivos. Martins et al. (1991b) investigaron sobre esto en siete "favelas" de Rio de Janeiro, en los años de 1984 y 1985. Schor (1990) realizó un estudio en el Hospital de Santo André (SãoPaulo), utilizando los datos obtenidos a partir de Historias Clínicas, identificando las características de naturaleza biológica, demográfica, social e institucional de 2.588 mujeres atendidas por complicaciones de aborto. Hardy \& Alves (1992) estudiaron los complicaciones post aborto provocado y los diversos factores asociados, planteando la necesidad de discutir en el seno de la sociedad este problema.

Un estudio de opinión respecto al aborto y su legalización fue realizado entre estudiantes de medicina y derecho de SãoPaulo, a través de cuestionarios, por Meira \& Ferraz (1989).

\section{Servicios de Salud, Relación Médico/Paciente}

Un análisis sobre el discurso médico ante la mujer, la construcción histórico-social del cuerpo y de la salud de la mujer y como éstos influyen en la relación médico/paciente es realizado por Borges (1991). Este mismo artículo fue presentado en la Conferencia Nacional de Salud y Derechos de la Mujer (1986).
La entrada de los médicos-parteros en la práctica obstétrica y el desplazamiento de las mujeres denominadas "comadres", que históricamente asistían a las mujeres en el trabajo de parto, pre-parto, abortos, enfermedades venéreas y otros aspectos de su salud, inaugura el encuadramiento del cuerpo femenino, como la producción de un saber anatómico y fisiológico de la mujer, a partir de la mirada masculina (Brenes, 1991).

En la búsqueda de alternativas para la atención a la mujer, Martins et al. (1991a) muestran el resultado de una experiencia de trabajo multidisciplinar y de evaluación de una práctica de atención en la consulta colectiva de ginecología; es el relato de una experiencia realizada en un centro de salud en la zona sur de Rio de Janeiro.

Aquino et al. (1986) evaluaron el estado en que se encuentra la detección precoz de cáncer de cuello uterino y la cobertura de la atención ginecológica en Brasil.

\section{Salud y Trabajo}

El campo de estudio de la salud de la mujer en el trabajo presentó pocas publicaciones, en este periodo. En la Conferencia se presentaron dos trabajos para la discusión; tanto Suarez (1986) como Dantas \& Silva (1986) dieron su atención al tema al referirse a los efectos del trabajo sobre la salud, condiciones de trabajo, distribución en el mercado de trabajo, efectos de la doble jornada; y sobre la salud de la mujer campesina, específicamente, en el estudio de Dantas \& Silva.

De los dos artículos que se publicaron en las revistas, uno es de orden teórico, señalando algunos aspectos de esta discusión y "procura mostrar a importância da abordagem do gênero na construção teórica da saúde do trabalhador, uma vez que homens e mulheres são expostos a condições de trabalho diferenciadas no processo produtivo" (Brito \& D'Acri, 1991: 201). El otro es un análisis de la participación de la mujer en el mercado de trabajo del sector salud en Brasil desde el año 1970 hasta 1980, por Machado (1986). 


\section{Otros}

Incluimos en esta sección artículos que plantean temas diversos que no creímos conveniente incluir en ninguno de las otros grupos temáticos. Se trata de dos artículos, uno denominado "A violência masculina é dirigida para Eva ou María?" de Noronha \& Daltro (1991), que se refiere a la violencia a que está expuesta la mujer, así como las diferencias entre las muertes y actos violentos distribuidos por género. Este estudio fue realizado en Bahia.

De igual forma, identidad femenina, sexualidad y construcción social de lo femenino fueron temas presentes en la discusión de la Conferencia, a través del artículo de Zanetti (1986); aparecieron en algunos de los trabajos ya referidos como parte del referencial de análisis y se plantea en forma específica en el trabajo de Giffin (1991), "Nosso corpo nos pertence: a dialética do biológico e do social", publicado en el número temático de "Mulher e Saúde” de los Cadernos de Saúde Pública.

\section{CONCLUSIONES}

Mostramos los artículos publicados sobre el tema de la mujer, en las tres publicaciones periódicas seleccionadas, que representan importantes sectores de opinión e instituciones en el área de la salud pública. Al organizar los artículos por área temática, observamos el predominio de estudios correspondientes a la salud reproductiva, la mitad de los artículos se refirieron al tema de embarazo, parto, puerperio, aborto, planificación familiar, anticoncepción, y derechos reproductivos, con una línea de análisis que privilegia los aspectos biológico-reproductivos en la mujer.

Estos trabajos mostraron diferentes aspectos de esta realidad: el aborto sigue siendo utilizado por las mujeres como salida ante el embarazo no deseado, realizado casi siempre en condiciones precarias y sin la atención médica requerida, significando una importante causa de muerte entre mujeres jóvenes. Por otro lado, la implantación de métodos anticonceptivos representa, en muchos casos, un riesgo para la salud de las mujeres que, sometidas a programas experimentales, observan sus cuerpos modificarse y sufrir efectos no esperados o no notificados a ellas previamente como posibilidad. Los anticonceptivos orales y la esterilización quirúrgica son los más utilizados, según muestran estos estudios. El alto número de cesáreas impresiona y preocupa a quienes observan el exceso de intervencionismo médico sobre la mujer.

Los estudios de morbilidad y mortalidad, así como los anteriores, contribuyen con el aporte de datos a elaborar un perfil de las condiciones en las cuales se encuentra la mujer brasileña, representando un avance en la medida que aportan elementos para una discusión que requiere datos concretos que muestran la realidad que se está tratando.

En algunos artículos fueron abordados aspectos psico-sociales; sin embargo, no encontramos estudios en las área de salud mental discutido en Brasília por Arilha Bezerra (1986), alcoholismo, suicidio, ni reflexiones sobre sexualidad, y referentes a la violencia sexual. Son frecuentes los estudios de las mujeres en «edad fértil», reafirmando el interés por la reproducción, principalmente, y dejando de lado la mujer en su niñez y su vejez como períodos de su vida. La identidad femenina con respecto a raza, ocupación, cultura es poco analizada existiendo escasos dados en algunos de los trabajos en forma parcial. Apesar de que fueron discutidos en la conferencia por Silva (1986) y Potiguara (1986). El área de la salud de la mujer trabajadora es un campo sobre el cual se comienza a tener interés y los textos publicados muestran la preocupación por esta discusión teórica.

Predominó el uso de métodos cuantitativos, sendo que, en algunos trabajos, fundamentalmente del área de anticoncepción y aborto, se utilizó la metodología cualitativa.

La Conferencia de Salud y Derechos de la Mujer marcó el inicio de un camino donde instituciones y sectores gubernamentales, junto a organizaciones no gubernamentales, gremiales, políticas, feministas entre otras, crearon un espacio para discutir asuntos de interés relacionados a la situación de la mujer. 
En el año 1986 muchas de las discusiones se plantearon, y los años que siguieron a este evento están sirviendo para constatar algunos de los mencionados problemas y proseguir en este camino de la investigación. Muchos aspectos tratados en la conferencia no fueron motivo de estudio publicados en las revistas analizadas.

Nos parece importante mencionar que no son estas las únicas iniciativas que estas instituciones han mostrado para con el tema en discusión. El libro publicado en 1989 por Associação Brasileira de Pós-Graduação em Saúde Coletiva - ABRASCO, "Mulher, Saúde e Sociedade no Brasil", organizado por María Eliana Labra, representó un esfuerzo por discutir sobre la salud de la mujer, mostrando aspectos de su origen histórico, con ayuda de la antropología y de la psicología, para mostrar algunas de las características de la mujer; datos estadísticos mostran su ubicación social y económica en la sociedad brasileña y, finalmente, una reflexión teórica sobre las políticas, programas y prácticas que afectan la salud de la mujer.

Creemos necesario estimular la discusión teórico-metodológica en torno al tema planteado, así como la permanente reflexión en los espacios destinados al debate dentro de la Salud Pública. En este sentido esperamos servir de orientación para futuros trabajos, mostrando una primera idea sobre las lineas editoriales y el tipo de publicaciones sobre la mujer que aparecieron en los últimos años en estas revistas.

\section{RESUMEN}

\section{MONTERO, C. A. Estudios sobre la Mujer en} Tres Revistas de Salud Pública. Cad. Saúde Públ., Rio de Janeiro, 11 (2): 305-313, abr/jun, 1995.

Este trabajo pretende observar la influencia que la "Conferência Nacional de Saúde e Direitos da Mulher", realizada en Brasília, en 1986, tuvo sobre las principales publicaciones periódicas del área de la salud pública de Brasil, respecto al tema de la mujer. Se muestran los artículos publicados en tres revistas del área, relacionados con la Salud de la Mujer; sin pretender realizar un análisis profundo del contenido de los artículos citados. Para ello, se organizaron por área temática los artículos publicados a partir de 1986 en las tres revistas: Revista de Saúde Pública (Faculdade de Saúde Pública, Universidade de São Paulo, Saúde em Debate (Centro Brasileiro de Estudos de Saúde), y Cadernos de Saúde Pública (Escola Nacional de Saúde Pública, Fundação Oswaldo Cruz). Se examinaron los artículos que tratan de la mujer, número de publicaciones y aspectos tratados en su contenido.

Palabras Clave: Salud de la Mujer; Salud Pública; Publicaciones Científicas

\section{REFERENCIAS BIBLIOGRAFICAS}

AQUINO, E. M. L.; CARVALHO, A. I.; FAERSTEIN, E. \& RIBERO, D. C. S., 1986. Situação atual da detecção precoce do câncer cérvico-uterino no Brasil. Cadernos de Saúde Pública, 2: 53-65.

AQUINO, E. M. L.; MENEZES, G. M.; AMOEDO, M. B. E. \& NOBRE, L. C. C., 1991. Mortalidade feminina no Brasil, sexo frágil ou sexo forte? Cadernos de Saúde Pública, 7: 174-189.

AQUINO, E. M. L.; MENEZES, G. M. S. \& AMOEDO, M. B., 1992. Gênero e saúde no Brasil: considerações a partir da pesquisa nacional por amostra de domicílios. Revista de Saúde Pública, 26: 195-202.

ARILHA, M. M. \& BEZERRA, B. C., 1986. A Saúde Mental da Mulher como Possibilidade de Criação. Conferência Nacional Saúde e Direitos da Mulher, Brasília, DF: CNDM. (Mimeo.)

BETTIOL, H.; BARBIERI, M. A.; GOMES, U. A.; WEN, L. Y.; RES, P. M.; CHIARATTI, T. M.; VASCONCELOS, V. \& YAMAWAKI, R. M., 1992. Atenção médica à gestação e ao parto de mães adolescentes. Cadernos de Saúde Pública, 8: 404-413.

BORGES, S. M., 1991. Proposta para uma relação: profissionais de saúde e mulheres. Cadernos de Saúde Pública, 7: 284-289.

BORUCHOVITCH, E., 1992. Fatores associados à não utilização de anticoncepcionais na adolescencia. Revista de Saúde Pública, 26: 437-443.

BRENES, A., 1991. História da parturição no Brasil, século XIX. Cadernos de Saúde Pública, 7: 135149. 
BRITO, J. \& D'ACRI, V., 1991. Referencial de análise para o estudo da relação trabalho mulher e saúde. Cadernos de Saúde Pública, 7: 201-214.

BRONFMAN, M. N. \& CASTRO, R., 1989. Discurso y práctica de la planificación familiar: el caso de América Latina. Revista Saúde em Debate, 25: 61-68.

CARVALHEIRO, C. D. G. \& MANQO, A. R. X., 1992. Mortalidade feminina no período reprodutivo, $\mathrm{cm}$ localidade urbana da região sudeste do Brasil. Evolução nos últimos 20 anos. Revista de Saúde Pública, 26: 239-245.

CNDM (Conselho Nacional dos Direitos da Mulher), 1986. Os Serviços de Saúde: o que as Mulheres Querem. Conferencia Nacional de Saúde e Direitos da Mulher, Brasília, DF: CNDM. (Mimeo.)

COSTA, S. H.; MARTINS, I. R.; PINTO, C. S. \& FREITAS, S. R. S., 1989. A Prática de planejamento familiar em mulheres de baixa renda no município de Rio de Janeiro. Cadernos de Saúde Pública, 5: 187-206.

DANTAS, M. B. \& SILVA, V. L., 1986. Saúde e Trabalho da Mulher Camponesa. Conferência Nacional Saúde e Direitos da Mulher, Brasília, DF: CNDM. (Mimeo.)

FAUNDES, A. \& CECATTI, J. G., 1991. A operação cesária no Brasil: incidência, tendências, causas, consequiências e propostas de ação. Cadernos de Saúde Pública, 7: 150-173.

FEITOSA, H. N.; MORON, A. F.; BORN, D.; ALMEIDA, P. A. M., 1991. Mortalidade materna por cardiopatia. Revista de Saúde Pública, 25: 443-451.

GARCIA, G. R., 1986. Contraceptivos: o Que Sabemos e o Que Não Sabemos. Conferencia Nacional Saúde e Direitos da Mulher, Brasília, DF: CNDM. (Mimeo.)

GIFFIN, K. M., 1991. Nosso corpo nos pertence: a dialética do biológico e do social. Cadernos de Saúde Pública, 7: 190-200.

GUERRA, E. M.; BARRETO, O. C. O.; VAZ, A. J. \& SILVEIRA, M. B., 1990. Prevalência de anemia em gestantes de primeira consulta em centros de saúde da área metropolitana, Brasil. Revista de Saúde Pública, 24: 380-386.

GULNAR, A. \& MENDONQA, S., 1993. Câncer na população feminina Brasileira. Revista de Saúde Pública, 27: 68-75.

HARDY, E. E.; PINOTTI, J. A.; OSIS, M. J. D. \& FAUNDES, A., 1990. Variáveis reprodutivas e riscos para doenças benignas de mama. Estudo caso-controle. Revista de Saúde Pública, 24: 387-393.
HARDY, E. E.; MORAES, T. M.; FAUNDES, A.; VERA, S. \& PINOTTI, J. A., 1991. Adequação do uso de pílula anticoncepcional entre mulheres unidas. Revista de Saúde Pública, 25: 96-102.

HARDY, E. \& ALVES, G., 1992. Complicações pós aborto provocado: fatores associados. Cadernos de Saúde Pública, 8: 454-458.

HUTTLY, S. R. A.; BARROS, S. A.; VICTORIA, C. G.; LOMBARDI, C. \& VAUGHAN, J. P., 1990. Gravidezes subsequentes: quem as tem e quem as quer? Observações num centro urbano da região sul do Brasil. Revista de Saúde Pública, 24: 212-216.

KOIFMAN, R. J.; QUINHOES, E. P.; MONTEIRO, G. T. R.; RODRIGUES, R. \& KOIFMAN, S., 1991. AIDS cm mulheres adultas no município do Rio de Janeiro. Cadernos de Saúde Pública, 7: 232-250.

KOIFMAN, S.; PAES, S. J. P.; OLIVEIRA, D. P.; VIANNA, N. F.; GIOVANINI, M. E.; CASTRO, M. L. O.; MOTA, L. G.; POYARES, J. A. M., DOMES, F. M. P. \& CASTRO, A. M. M., 1987. Avaliação do anticoncepcional Norplant no municipio do Rio de Janeiro, RJ (Brasil). Revista de Saúde Pública, 21: 513-522.

LABRA, M. E. (Org.), 1989. Apresentação. In: Mulher, Saúde e Sociedade no Brasil, pp. 13-21, Petrópolis: Vozes/Rio de Janeiro: Abrasco. (Coleção Saúde e Realidade Brasileira)

LIPPI, U. G.; ANDRADE, S. A.; BERTAGNON, J. R. D. \& MELO, E.,1989. Fatores obstétricos associados ao baixo peso ao nascer. Revista de Saúde Pública, 24: 382-387.

LOLIO, C. A.; LAURENTI, R.; BUCHALA, C. M.; SANTOS, A. H. \& JORGE, M. H. P. M., 1991. Mortalidade de mulheres em idade fértil no município de São Paulo (Brasil), 1986. Mortes por diferentes causas: doenças cardiovasculares. Revista de Saúde Pública, 25: 37-40.

LOPEZ, F. V. A.; SCHOR, N. \& SIQUEIRA, A. A. F., 1989. Gravidez na adolescência: estudo comparativo. Revista de Saúde Pública, 23: 473-477.

MACHADO, M. E., 1986. A participação da mulher no setor saúde no Brasil - 1970/80. Cadernos de Saúde Pública, 2: 449-465.

MARTINS, D. M.; AMARAL, J. L.; DOURADO, M. T. L. \& DOMES, N. C. C., 1991 a. Consulta coletiva: o espaço da mulher. Cadernos de Saúde Pública, 7: 267-283.

MARTINS, I. R.; COSTA, S. H.; FREITAS, S. R. S. \& PINTO, C. S., 1991b. Aborto induzido cm mulheres de baixa renda: dimensão de um problema. Cadernos de Saúde Pública, 7: 251-266. 
MEIRA, A. R. \& FERRAZ, F. R. C., 1989. Liberação do aborto: opinião de estudantes de medicina e de direito, São Paulo. Revista de Saúde Pública, 23: 465-472.

MS (Ministério da Saúde)/INAN (Instituto nacional de Alimentação e Nutrição), 1983. Assistência Integral à Saúde da Mulher. Bases Para Uma Prática Educativa. Documento Preliminar 1986. Brasília, DF: MS.

NORONHA, C. \& DALTRO, M. E., 1991. A violência masculina é dirigida para Eva ou Maria? Cadernos de Saúde Pública, 7: 215-231.

POTIGUARA, E., 1986. A Identidade da Mulher Índia. Conferência Nacional Saúde e Direitos da Mulher, Brasília, DF: CNDM. (Mimeo.)

RAVELO, P., 1990. La salud de la mujer desde la perspectiva antropológica: una revisión preliminar. Revista Salud Problema, 20: 39-29.

REIS, A. R., 1986. Direitos da Reprodução Humana. Os Herdeiros da Terra. Uma Reflexão Sobre a Procriação e o Direito. Conferência Nacional Saúde e Direitos da Mulher, Brasília: CNDM. (Mimeo.)

SCHOR, N., 1990. Investigação sobre ocorrência de aborto em pacientes de hospital do centro urbano do estado de São Paulo, Brasil. Revista de Saúde Pública, 24: 144-151.

SCHOR, N. \& LOPEZ, A. F., 1990. Adolescência e anticoncepção: estudo de conhecimento e uso em puérperas internadas por parto ou aborto. Revista de Saúde Pública, 24: 506-511.
SILVA, B., 1986. A Identidade da Mulher Negra - A Identidade da Mulher Índia. Conferência Nacional Saúde e Direitos da Mulher, Brasília, DF: CNDM. (Mimeo.)

SILVA, K. S., 1992. Mortalidade materna: avaliação da situação no Rio de Janeiro, no período de 1977-1987. Cadernos de Saúde Pública, 8: 442-453.

SIQUEIRA, A. A. F.; SANTOS, J. L. F. \& SILVA, J. F., 1986. Relação entre estado nutricional da gestante, fumo durante a gravidez, crescimento fetal e no primeiro ano de vida. Revista de Saúde Pública, 20: 421-434.

SUAREZ, M., 1986. Mulher, Trabalho e Saúde. Elementos para Discussão. Conferência Nacional Saúde e Direitos da Mulher, Brasília: CNDM. (Mimeo.)

TAKIUTI, A., 1986. A Mulher Adolescente: Urna Abordagem. Conferência Nacional Saúde e Direitos da Mulher, Brasília, DF: CNDM. (Mimeo.)

TANAKA, A. C. d'A.; SIQUEIRA, A. A. F. \& BAFILE, P. N., 1989. Situação de saúde materna e perinatal no estado de São Paulo, Brasil. Revista de Saúde Pública, 23: 67-75.

ZANETTI, M., 1986. A Identidade Feminina. Conferência Nacional Saúde e Direitos da Mulher, Brasilia, DF: CNDM. (Mimeo.) 